\title{
LA PERSPECTIVA DE GÉNERO EN LOS PROGRAMAS DE ESTUDIO DE LAS LICENCIATURAS CONTABLES ADMINISTRATIVAS
}

\author{
The gender perspective in the curriculum \\ of management accountants degrees
}

María Elena Camarena Adame ${ }^{1}$, María Luisa Saavedra García ${ }^{2}$

Fecha de recepción: 15 de agosto de 2016

Fecha de aceptación: 04 de octubre de 2016

1- Nacionalidad: Mexicana. Grado: Doctorado en administración. Especialización: Factor humano, cultura y género en las organizaciones. Adscripción: Universidad Nacional Autónoma de México. Correo electrónico: mcamarena_adame@hotmail.com

2- Nacionalidad: Mexicana. Grado: Doctora en administración. Especialización: Finanzas corporativas. Adscripción: Universidad Nacional Autónoma de México. Correo electrónico: maluisasaavedra@yahoo.com 


\section{Resumen}

La perspectiva de género consiste en visualizar los distintos fenómenos de la realidad tomando en cuenta que en todos los ámbitos existen diferencias entre el actuar de hombres y mujeres. El objetivo de este trabajo consiste en realizar una propuesta para incorporar la perspectiva de género en el currículo de los programas de estudio de las licenciaturas contables administrativas, para lo cual se realiza un análisis documental con el fin de comprender en primer lugar las temáticas: Universidad y género, el género en el currículo universitario, La perspectiva de género en el Plan Nacional de Desarrollo y en el Programa Sectorial de Educación en México y los estudios de género en las organizaciones, posteriormente se realiza una revisión de las publicaciones realizadas en México que abordan la temática de estudios de género en las organizaciones, clasificando por líneas de investigación los referidos trabajos, por último se presentan las temáticas que podrían incorporar los programas de estudios de estas disciplinas.

Palabras clave: Enseñanza aprendizaje, género, currículo.

\section{Abstract}

The gender perspective involves visualizing the phenomena of reality in the context of differences between men and women in all areas. The aim of the present paper is to propose incorporating the gender perspective in the curriculum of business administration professional degrees. An analysis of the relevant literature led to understanding such issues as university and gender, gender in the university curriculum, gender perspective in national development plans including education in Mexico and gender studies in organizations. A review of relevant publications in Mexico addressing the issue of gender studies in organizations, led to classifying them by lines of research. Finally the issues that should be added to the curricula of these disciplines are presented.

Keywords: Teaching learning, gender, curriculum development. 


\section{Introducción}

$\mathrm{L}$

os expertos en género ${ }^{3}$ f feminismo ${ }^{4}$ están trabajando desde hace décadas en la comprensión de las relaciones de dominación entre hombres y mujeres en sus múltiples manifestaciones. trucción de los Estados sin la participación de la mitad de la sociedad (que son las mujeres) o con una mínima representación de ellas, a la feminización de la pobreza (los estadistas señalan que la pobreza tiene cara de mujer), ese paradigma de explotación económica que es la maquila, sobre la violencia de género, desde el feminicidio hasta otras nuevas formas de violencia patriarcal, los efectos nocivos de las políticas neoliberales sobre la vida de las mujeres, sobre la politización del marco doméstico y el aumento del trabajo gratuito que realizan en el hogar, sobre los cambios que se están produciendo en la familia patriarcal o sobre los déficits de autonomía personal de las mujeres y cómo este hecho dificulta su capacidad de negociación en la vida familiar con los varones, entre otros temas (Molina y Nava, 2009: 51).

Las investigaciones acerca de la problemática del género en las organizaciones han sido incipientes en México, a pesar que persiste una marcada desigualdad como consecuencia entre otros aspectos del "techo de cristal", que impide a las mujeres ascender a puestos de alto nivel, limitando de este modo su desarrollo (Camarena y Saavedra, 2015b: 76, Camarena, Saavedra y Ducloux, 2015c: 4). En este sentido Vázquez (2013: 1) señala que en México aún son escasos los estudios encaminados a impulsar con suficiente autonomía la perspectiva de género dentro de la administración.

La importancia de impulsar los estudios de género consiste en que estos son una herramienta esencial para integrar de manera eficaz el enfoque de género en políticas, programas, planes y proyectos de desarrollo $^{5}$; así como, la transversalidad de género ${ }^{6}$ y el empoderamiento de la mujer (Molina y Nava, 2009: 120). Por otra parte, Navarro (2000:33) ha señalado que organizaciones desequilibradas en cuanto al género producen impactos también desequilibrados, por lo que es necesario promover que más mujeres participen en la toma de decisiones.

Las cifras muestran el importante aporte de las mujeres a las economías de sus hogares cuando están recibiendo un ingreso por su trabajo. Sin embargo, este podría ser mucho mayor si su inserción laboral no revistiera la precariedad que caracteriza a un gran porcentaje de ocupaciones desempeñadas por mujeres en trabajos desprotegidos, en los sectores de menor prestigio y remuneraciones de la economía; más aún, si se elimina la brecha aun sensible entre ingresos de hombres y mujeres que realizan un trabajo igual o similar (CIM, 2011: 13; Heller, 2010: 31).

3- Es el conjunto de características de personalidad, gustos, preferencias, actitudes, papeles y valores que desarrolla una persona cuando se identifica con un sexo (Serret y Méndez, 2011: 12).

4- El feminismo se caracteriza por la demanda de derechos para las mujeres, pensando que al obtenerlos se lograría la igualdad con los hombres (Serret y Méndez, 2011: 14).

5- Aunque como señala González (2009) el término "género" es ya muy popular entre políticos, medios de comunicación y buena parte de la población, que lo utilizan con tremenda vaguedad (se asocia con mujeres o con derechos de mujeres).

6- Se conoce también como "enfoque integrado de género" y pretende asegurar que a través de las políticas públicas y privadas se promueven los cambios estructurales necesarios para eliminar la desigualdad entre mujeres y hombres a largo plazo, equilibrando la posición entre ambos, y asegurando el pleno disfrute de derechos y el acceso equitativo a los recursos (OIT, 2011: 6). 
En México, INMUJERES es una institución del gobierno federal que goza de autonomía en sus funciones técnicas y gerenciales. Financiada por el Banco Mundial, INMUJERES está implementando GENEROSIDAD, un programa nacional de certificaciones de igualdad de géneros inspirado en el Modelo de Equidad de Género de 2003. Este modelo promueve estándares nacionales comerciales equitativos en la remuneración, el desarrollo profesional, las oportunidades de capacitación y otros aspectos de gestión (Piazze, 2009: 5). Por su parte, el PNUD (Programa de las Naciones Unidas para el Desarrollo) ha lanzado la metodología COpEQ (Cambio Organizacional pro Equidad) que podría ser catalogada como un tipo de investigación-acción que propicia que se revise el funcionamiento organizacional y se identifiquen aquellos aspectos que se quieren cambiar, al considerarse que propician inequidades (Navarro, 2007: 21).

De acuerdo con Serret (2008: 47) la perspectiva de género se ha incorporado en los ámbitos políticos y académicos en México; se ha empezado por emplear un lenguaje políticamente correcto, que evita la exclusión de las mujeres, incorporando temas acerca de la subordinación femenina en sus agendas políticas.

Lo anterior, destaca la importancia de realizar una propuesta para incorporar la perspectiva de género en las organizaciones en el currículo de los programas de licenciatura de las ciencias económico administrativas, para lograr este objetivo en primer lugar se realiza una descripción acerca de los estudios de género en las organizaciones encontrando la forma como la disciplina administrativa se ha desarrollado históricamente con un enfoque androcéntrico, posteriormente se realiza un análisis de las investigaciones que se han presentado en los dos congresos más importantes en Ciencias Administrativas en México, abarcando un periodo de cinco años, encontrando la notoria preocupación que existe por parte del medio académico, acerca de la inequidad de género que existe en las organizaciones, por último se realiza una propuesta de las principales temáticas que deberían ser incorporadas en el currículo de los programas de las licenciaturas en ciencias contables administrativas.

\section{Universidad y género}

A lo largo de los años ochenta surgió un importante número de programas de estudios de la mujer y de género en universidades e instituciones de educación superior de América Latina y, particularmente, de México; en los noventa aumentan los estudios de género y éste tema se institucionaliza en las universidades, por lo que se crean cursos, programas, centros y áreas en relación al tema hasta llegar a la creación de redes. En cuanto a las investigaciones alrededor del tema sobresalen estudios acerca de las académicas universitarias como generadoras de conocimientos y aportes a la ciencia (Cardaci, 2005: 110), reconociéndolas como portadoras y transmisoras de sabiduría y conocimiento (Rivera, 2003: 5).

Se puede encontrar una evolución completa de ese fenómeno en Cardaci (2005: 121), así tenemos que entre 1960 y 1982 se dio en México el periodo más dinámico de expansión y diferenciación de la educación superior en el periodo 1970-1998, el crecimiento de la matrícula femenina fue de 256\%, en tanto que la masculina creció solamente en $60 \%$; en los años siguientes la distribución porcentual continuó su tendencia hacia la nivelación entre los dos sexos llegando a ser en 2001 de $52.5 \%$ para hombres y $47.8 \%$ para mujeres (Cardaci, 2005: 135). 
Los datos que se presentan a continuación se obtuvieron mediante una encuesta que se aplicó a integrantes de centros y programas de estudios de la mujer y de género y a informantes claves (coordinadores/as de licenciaturas, docentes, investigadores/as) en instituciones de educación superior (Cardaci, 2005: 137):

- Se identificó un total de 41 unidades de enseñanza-aprendizaje que han incorporado contenidos sobre la condición de las mujeres y/o sobre las relaciones de género en el currículo de quince licenciaturas que abordan diversos campos de estudio.

- En el área de educación y humanidades, que tenía $66.1 \%$ de matrícula femenina en 2001, se localizó el mayor número de cursos. La licenciatura en psicología concentra seis, historia cuatro, filosofía tres, antropología tres, pedagogía dos, etnología dos y letras uno.

- No se reportaron cursos en contaduría o administración, licenciaturas que cuentan con una matrícula femenina de $58.2 \%$ y $56.03 \%$, respectivamente.

- No se identificaron cursos que se ofreciesen en licenciaturas propias de las ciencias agropecuarias, ciencias exactas y naturales o ingeniería y tecnología.

Los hallazgos de la investigación de Cardaci (2005: 137) dan cuenta de todo lo que falta por hacer para incorporar los estudios de género en el currículo universitario, aunque ya desde hace tiempo se han hecho esfuerzos para que esto suceda. Como un referente importante en México, se tiene el caso de la Universidad Nacional Autónoma de México (UNAM), el proceso de incorporación de materias con perspectiva de género empezó en los años setenta, en la Facultad de Ciencias Políticas y Sociales, en los años ochenta le siguieron las carreras de psicología y enfermería, y en algunos posgrados de psicología (Buquet, 2011: 213). Sin embargo, es necesario resaltar que la incorporación de la perspectiva de género en la enseñanza de nivel superior, hasta la fecha no ha logrado implementarse de manera formal y definitiva y tampoco en todos los planes de estudio (Buquet, 2011:214).

La institucionalización de los estudios de género en la UNAM se logró en 1992 a través de la implementación del Programa Universitario de Estudios de Género (PUEG), por una propuesta que surgió en 1990 en el Congreso Universitario en la mesa I. Universidad y Sociedad; el PUEG ha aportado una riqueza notable en el ámbito de la investigación y la difusión de estudios de género, realizando estudios cuantitativos y cualitativos que ponen de manifiesto ciertas tendencias de segregación en las poblaciones académica, administrativa y estudiantil, así como los factores que influyen en la perpetuación de estas desigualdades (Buquet, 2011: 220).

\section{El género en el curriculum universitario}

Marinsalta (2010) señala que se deben modificar profundamente los hábitos educativos con el fin de renovar el currículo mediante la transversalización de la categoría de género. Pues sostiene que el modelo de currículo actual está fundamentado en supuestos androcéntricos manifestado en los conceptos, metodologías y tácticas educativas, trayendo como consecuencia que las mujeres estén ausentes y silenciadas. Como solución sugiere recuperar y revalorizar las contribuciones femeninas con el fin de eliminar 
los esquemas y formas de producción que la ideología dominante recrea a través de los tradicionales estereotipos sociales.

Por lo tanto, de acuerdo con Bolaños (2003: 72) un currículo universitario de género sensitivo e inclusivo será aquel que propicie la reflexión crítica de la sociedad y que por consiguiente, considere las relaciones de género y características del sistema patriarcal imperante, con la finalidad de generar procesos transformadores de dichas relaciones y con ello la construcción de una sociedad equitativa, justa y pacífica. Como proceso histórico, de negociación/ imposición, en la construcción de un currículo universitario para la formación de profesionales en una determinada disciplina, todos los integrantes de una unidad académica, juegan un papel central, al aportar sus propias visiones de mundo, sus creencias, es un proceso participativo y reflexivo, pues en esto radica principalmente, la posibilidad de conocer ampliamente la profesión y la disciplina para concretar la selección y organización de los contenidos curriculares. Por lo anterior, es primordial la construcción de un marco socio-histórico de la profesión y de un marco epistemológico de la disciplina. Estableciendo así los propósitos de una carrera para realizar la selección de contenidos y establecer la secuencia y organización de estos en el plan de estudio, así como las estrategias de ejecución, seguimiento, evaluación y administración curricular. Es en la elaboración de estos dos marcos donde se debería introducir la perspectiva de género, pues a través del análisis del contexto histórico-político-social de la profesión, de las prácticas profesionales, del desarrollo de la disciplina, de la forma en la cual se construye el conocimiento, de quienes conforman la comunidad científica en dicha profesión, de los aspectos éticos de la disciplina, con base en los elementos teóricos y metodológicos que aporta la teoría de género y el paradigma cultural del humanismo, se debe trascender de la simple inclusión del asunto de género, como un tema o curso simplemente, a la concepción de esta problemática de una manera integral.

Por su parte, Velasco (2007: 47) propone incorporar el enfoque de equidad de género en los currículos, así como en planes y programas de estudio, en las diferentes áreas del conocimiento; atender la transformación del currículo oculto, discriminatorio, reflejado en lenguaje, actitudes y gustos; llevar a cabo programas de orientación no sexistas con el fin de ampliar los criterios de selección de carrera impulsando la participación de mujeres y hombres en todas las áreas del conocimiento.

Maceira (2005: 192) sugiere que el currículo oculto se puede constituir en un medio para introducir el enfoque de género en las Instituciones de Educación Superior, dado que en toda institución educativa existe un "currículo oculto de género", es decir, una serie de valoraciones, códigos, normas, ideas, supuestos, mitos, discursos, conceptos, creencias, relaciones de poder, roles, recursos textuales y simbólicos generalizados y sexistas que se transmiten en la escuela y que refuerzan la discriminación femenina de manera poco visible. Por lo que, propone la investigación, explicitación y transformación de los currículos ocultos de género como uno de los recursos fundamentales del proceso complejo de incorporación de la perspectiva de género en las instituciones de educación superior dado que permite conocer los mecanismos de violencia, discriminación y desigualdad y proponer acciones para cambiarlos.

Caballero (2011:59) reconoce que incluir la perspectiva de género en el currículo representa un gran reto para la elaboración de planes y programas de estudios de las Instituciones de Educación Superior (IES), dado que se deben incluir prácticas y contenidos encaminados a erradicar la inequidad entre los sexos en la vida escolar. A este respecto sugiere que utilizar la transversalización no siempre es garantía de la inclusión de la perspectiva de género en el currículo, pues resulta insuficiente para modificar de 
fondo la inequidad entre los sexos y asegurar la igualdad de oportunidades a hombres y mujeres. Aunque Flores, Carrazco y Espejel (2014: 25) sugieren que se puede tomar como un comienzo siendo útil para empezar atender las necesidades de las mujeres y de los hombres que quieran incluirse, fomentando la unificación para no hacer de esta lucha un andar aislado.

La incorporación de la perspectiva de género en el currículo universitario también, ha sido propuesta por Durán (2012: 40) quien señala que se debe tomar en cuenta: el análisis e incorporación del enfoque de género en el currículo, planes y programas de estudio; creación de cursos específicos, temas y contenidos sobre los estudios de género tanto a nivel de pregrado (técnico y diplomado), grado y posgrado de las diferentes áreas del conocimiento.

Por último, Buquet (2011: 221) establece que la incorporación de los estudios de género en los currículos es un factor que favorece el proceso de institucionalización de la perspectiva de género en las IES, y se enfoca a dos objetivos complementarios: 1) Tiene un impacto directo en la preparación académica de los jóvenes, proporcionándoles elementos para comprender la realidad social en este tópico; 2) La discusión de los temas con perspectiva de género, aporta a la formación de los jóvenes universitarios elementos para la deconstrucción de las diversas formas de discriminación imperantes en la sociedad y les transmite valores de equidad y respeto a las diferencias.

\section{La perspectiva de género en el Plan Nacional de Desarrollo y en el Programa Sectorial de Educación en México}

Las acciones del Gobierno respecto a la perspectiva de género, se encuentran enunciadas en el Plan Nacional de Desarrollo 2013- 2018: 165, el cual señala:

Perspectiva de género. La presente administración considera fundamental garantizar la igualdad sustantiva de oportunidades entre mujeres y hombres. Es inconcebible aspirar a llevar a México hacia su máximo potencial cuando más de la mitad de su población se enfrenta a brechas de género en todos los ámbitos. Éste es el primer Plan Nacional de Desarrollo que incorpora una perspectiva de género como principio esencial. Es decir, que contempla la necesidad de realizar acciones especiales orientadas a garantizar los derechos de las mujeres y evitar que las diferencias de género sean causa de desigualdad, exclusión o discriminación.

El objetivo es fomentar un proceso de cambio profundo que comience en las instituciones de gobierno. Lo anterior, con el objeto de evitar que en las dependencias de la administración pública federal se reproduzcan los roles y estereotipos de género que inciden en la desigualdad, la exclusión y discriminación, los cuales repercuten negativamente en el éxito de las políticas públicas. De esta manera, el Estado mexicano hará tangibles los compromisos asumidos al ratificar la Convención sobre la Eliminación de todas las Formas de Discriminación contra la Mujer (CEDAW, por sus siglas en inglés), así como lo establecido en los artículos 2, 9 y 14 de la Ley de Planeación, referentes a la incorporación de la perspectiva de género en la planeación nacional.

En este sentido, el Programa Sectorial de Educación (PSE) 2013-2018, sección III.2, estrategia 2, señala "Impulsar la perspectiva de género y de derechos humanos en los procesos de planeación y evaluación del sector educativo“ p. 73, para instrumentar esta estrategia en la línea de acción 2, propone la incor- 
poración en los planes y materiales de estudio las perspectivas de igualdad entre mujeres y hombres, de derechos humanos y de no discriminación, con el propósito de eliminar los estereotipos de género y prevenir los actos de violencia contra las mujeres.

Bajo este contexto se entiende que la educación debe lograr que hombres y mujeres accedan a un conjunto de saberes, habilidades y valores que les permitan estar en mejores y más equitativas condiciones. Hay que ofrecer una educación que posibilite el respeto mutuo entre hombres y mujeres, que legitimen y valoricen el conjunto de necesidades fundamentales, entre ellas la afectividad, la libertad, la autoestima, la participación y que rechacen cualquier discurso o práctica discriminatoria (Camarena, Saavedra y Ducloux, 2015: 21).

\section{Estudios de género en las organizaciones}

Los estudios de género en las organizaciones analizan el comportamiento de hombres y mujeres dentro de los roles que desempeñan en el contexto de las organizaciones. Comprender las acciones de los varones como el sexo dominante en las organizaciones modernas, exige comprender cómo se construyen en el trabajo las masculinidades que excluyen, en primer lugar, a la mujer de puestos clave de decisión y en segundo lugar a minorías como indígenas y homosexuales, implica también identificar cómo se establecen los nexos significativos entre los espacios laborales e individuos sexuados, para tener la posibilidad de delinear patrones de comportamiento propios. Es así como la incorporación de la perspectiva de género, permite saldar una tarea pendiente, ya que forma parte de la agenda en el análisis organizacional contemporáneo (Vázquez, 2013: 4).

Bajo este concepto, las relaciones de género son relaciones de poder ${ }^{7}$. La forma de actuar dentro de las organizaciones está saturada de fuerzas más o menos explícitas. El ejercicio profesional en el contexto de las organizaciones tiene que ver con el género de sus integrantes.

Mujeres y hombres enfocan su desarrollo y promoción profesional de manera diferente. Frecuentemente ellas se retiran de esa escalada cuando se casan, para atender a los hijos, por la convicción de que valen menos, por falta de estímulos externos, por reproches del medio familiar y social, por culpabilidad, por el tremendo esfuerzo que se les exige, porque tienen que competir con personas en mejores condiciones, y por ello su trabajo se remunera menos.

Además, a los hombres no les gusta estar supeditados a mujeres. Ellas mismas los eligen para gobernar en un significativo gesto de sumisión. Una profesión es para el hombre lo que es la maternidad para la mujer.

Resulta significativo que la presencia más numerosa y el éxito más claro de las mujeres en las diversas etapas de su desarrollo, sufre un quiebre en tres momentos de excepcional importancia (Santos, 1997: 22):

7- Estas relaciones son una expresión del androcentrismo, enmarcado dentro del patriarcado. El androcentrismo sitúa la mirada masculina en el centro del universo, como medida de todas las cosas y representación global de la humanidad, ocultando otras realidades, entre ellas la de la mujer. De acuerdo con Serret (2008: 3) en todas las sociedades estas relaciones se traducen en la subordinación de las mujeres a los hombres. 
a) La elección de carrera: Los estudios de mayor relevancia social y económica tienen mayor presencia de varones, frente al número más elevado de mujeres en las carreras de carácter social (Enfermería, Magisterio, Psicología, Pedagogía, Psicopedagogía, Humanidades, etc.). Por lo tanto, el género es un factor diferenciador en la elección vocacional y uno de sus condicionantes (Mosteiro, 1997: 311).

Las expectativas de los padres y madres sobre el futuro profesional de las hijas son menores que las que tienen respecto a los hijos. Los profesores y profesoras manifiestan menor apoyo y potencian menos a las niñas en sus opciones profesionales. Las mismas niñas se consideran incapacitadas para algunas materias o profesiones, encarnando la profecía de autocumplimiento de la que habla Watzlawick ${ }^{8}$.

b) La incorporación al mundo del trabajo: Pese a la manifiesta e inequívoca demostración de capacidades de las mujeres en el sistema educativo, el acceso al mundo del trabajo está claramente marcado por la discriminación. Las causas tienen aquí un componente social importante (los patrones ejercen una sutil o burda discriminación) y también de carácter psicológico (en caso de que uno de los dos miembros deba atender al hogar y los hijos, es la mujer la que asume este papel de forma voluntaria u obligada). Jiménez y Beltrán (2015: 12) también señalan que la masculinización de las organizaciones se debe a que la mujer llegó mucho después que el hombre al mercado laboral.

c) El desarrollo profesional: Muchas mujeres, guiadas por patrones profesionales, recortan o limitan su futuro profesional bajo diversas excusas (atención del hogar, cuidado de los hijos, cesión de sus derechos a los intereses del marido, presiones sociales, etc.).

Todo ello tiene que ver con la percepción de cada mujer y además tiene influencia en otras mujeres que se guían por los modelos establecidos. De ahí la importancia de cambiar la formación, las actitudes, las concepciones y las prácticas.

A pesar de que la presencia de la mujer en el mundo laboral, sobretodo en sus niveles inferiores, es igual a los hombres, es reducido el número de mujeres que ocupan cargos directivos.

La razón real de la ausencia de la mujer en tareas de dirección se explica por su falta de identificación con el modelo de liderazgo imperante, y a las diferencias de modos, maneras y estilos en relación a sus compañeros directivos, evidentemente al margen de la eficacia de un estilo u otro en la gestión (Santos, 1994 :9). De acuerdo con Moreira (2010: 51) las diferencias de estilo entre hombres y mujeres y los estereotipos de género en el dominio del liderazgo pueden explicarse por dos fenómenos: en primer lugar, hay diferencias en el comportamiento exhibido por los líderes hombres y mujeres y, en segundo lugar, pueden existir estereotipos asociados a un liderazgo eficaz, lo que impide que muchas mujeres se consideren buenos líderes. Sin embargo, dos hitos han marcado el ingreso de la mujer a puestos de toma de decisiones: uno el incremento en los niveles de escolaridad y dos, cambios en la manera en que la sociedad percibe el trabajo femenino; pero a pesar de esto las mujeres todavía se ven obligadas a aceptar

8- Watzlawick, decía que la profecía de un suceso lleva al cumplimiento de la profecía, ver: Watzlawick (1989: 2). 
sueldos y condiciones de trabajo poco equitativos con relación a los que perciben los hombres en los mismos puestos (Camarena y Hernández, 2005: 3).

Aunque actualmente, las mujeres han aumentado su participación en las actividades administrativas y de gestión, se suele ubicar a las mujeres en los niveles de dirección que corresponden a sectores no estratégicos y cargos de personal administrativo y no así en puestos direccionados hacia cargos ejecutivos que conducen a la alta dirección. Estas desventajas se acentúan por el hecho de que las mujeres quedan excluidas de las redes tanto formales como informales, las cuales son imprescindibles para la promoción profesional en la empresa (Ojeda y Avilés, 2005: 9).

\section{¿Por qué resulta tan difícil alcanzar la equidad de género dentro de organizaciones?}

Es sorprendente que en un sistema socioeconómico en el que se profesa 'el individualismo meritocrático, existan todavía tan pocas mujeres en posiciones de poder y liderazgo, puesto que la carrera profesional la construye cada persona dependiendo del esfuerzo que esté dispuesta a invertir en su empeño. Una manera de encontrar la respuesta a esta problemática se puede plantear a través de preguntas acerca de las fuentes de las desigualdades que vemos, sentimos, vivimos y de los tipos de poder que las nutren y mantienen (Navarro, 2003: 11).

Así pues, los cuestionamientos de acuerdo con Navarro (2003: 17) serían ¿Quiénes ejercen el poder?, ¿Cómo lo ejercen?, ¿Cómo podría distribuirse? La respuesta a estas preguntas se puede encontrar analizando las estructuras profundas que en cada organización propician fórmulas de distribución de poder particulares y, por tanto, de inequidad de género. Posteriormente, a partir de la identificación de los diferentes tipos y dimensiones de poder presentes en la organización, analizar cómo éstos inciden en las dinámicas de participación/exclusión para generar aportes que posibiliten relaciones democráticas de poder basadas en la equidad de género.

Las organizaciones modernas representan un excelente pretexto para debatir cómo mujeres y varones interactúan en estos espacios sociales donde para millones de personas del mundo occidental representan más que su centro laboral (Vázquez, 2013: 2). Los varones y mujeres que se integran a las organizaciones como fuerza laboral, lo hacen bajo estas condiciones estructurales, así como también en un nivel de micro interacción donde portan y reproducen su forma de socialización alrededor de su concepción de género.

\section{Las líneas de investigación de estudio de género en las organizaciones en México}

Tradicionalmente, la perspectiva de género en el terreno de la administración se ha enfocado en analizar los procesos sociales de una manera usualmente no abordada hasta la década de los setenta del siglo pasado: la mujer en el trabajo (Vázquez, 2013: 6).

Los estudios de género en las organizaciones principalmente se orientan a una tradición teórica anclada en las perspectivas feministas, que visibilizan la discriminación de la mujer para escalar posiciones jerárquicas superiores en las grandes empresas, o que limitan su avance como empresaria (Techo de 
cristal $^{9}$, techo de cemento ${ }^{10}$, suelo pegajoso ${ }^{11}$ ). A este respecto, Alvesson y Billing (citados en Vázquez, 2013: 11) señalan que existen dos grandes discusiones, la primera descubre un complejo tejido de relaciones entre los varones, donde la mujer no es tomada en cuenta, pues mientras que la mujer debe demostrar frente a otros varones que cuenta con las capacidades para competir y ocupar puestos gerenciales, los varones cuentan con mecanismos preestablecidos que facilitan su ascenso, como redes informales integradas por ellos mismos dejando a la mujer en absoluta desigualdad. La segunda discusión, tiene que ver con la expresión en las relaciones de desigualdad entre varones y mujeres, alrededor de la conformación hegemónica de la masculinidad en el trabajo (Vázquez, 2013: 14). Lo anterior implica que en el mundo empresarial los puestos de poder y liderazgo se encuentran configurados únicamente con rasgos masculinos.

Vázquez (2013:6) ha propuesto temáticas que pueden conformarse en líneas de investigación en las organizaciones mexicanas con perspectiva de género, estas son:

La toma de decisiones en la organización. El género aparece como un aspecto a tomarse en cuenta para la realización de diagnósticos organizacionales que buscan modificar aspectos tan técnicos, como la aplicación de planes estratégicos, considerando que son los varones quienes dominan la toma de decisiones en las organizaciones. Sin embargo, hombres y mujeres actúan de modo diferente al momento de tomar una decisión, p.e. al evaluar candidatos para un puesto, el reclutador preferirá al mejor calificado y con más experiencia y habilidad en el puesto, caso contrario, la reclutadora se deja llevar por los sentimientos y podría contratar al que más necesidad tiene del trabajo.

Participación de la mujer para ascender a puestos de decisión clave. Trata del estudio de cómo las mujeres ejecutivas son objeto de inequidades y discriminación para ascender de puestos gerenciales. Y tiene que ver con el techo de cristal, es decir con las barreras que existen en todas las organizaciones y que pueden ser de carácter, social, cultural o de otra índole, que impiden que la mujer ascienda a puestos de alta dirección y si asciende, su participación es únicamente representativa y no se le remunera igual que al hombre.

Configuración de la cultura organizacional. La identificación de los principales rasgos culturales en una organización ayuda a reconocer que en toda organización coexiste una red de significados junto con las estructuras formales, es aquí donde la perspectiva de género requiere posicionarse. Todo alrededor de las organizaciones se encuentra masculinizado, no se toma en cuenta que la mujer tiene modos diferentes de pensar, actuar, etc., por lo que se tiene que generar una cultura de equidad que reconozca estas diferencias.

Reconocimiento de una hegemonía masculina en la dinámica organizacional. La literatura en torno a la administración da cuenta de modelo analítico hegemónico de organizaciones y ejercicio de la administración, con una clara dominación masculina (Jiménez y Beltrán, 2015: 8). De donde se desprenden anarquías organizadas que caracterizan al tomador de decisiones, varones que despliegan diversas estrategias, es decir temas importantes en donde no ha sido incorporada la perspectiva de género. En la

9- El techo de cristal son los obstáculos que impiden a las mujeres alcanzar puestos de alto nivel en las organizaciones.

10- El techo de cemento son las limitaciones que se ponen las empresarias para su crecimiento, como respuesta a mantener un equilibrio entre el trabajo y la familia.

11- El suelo pegajoso es el que mantiene a las mujeres en puestos de bajo nivel e importancia y de menor salario. 
- Revista de Ciencias Sociales y Humanidades. ISSN-P: 0188-9834 ISSN-E: 2395-8669.

literatura sobre gestión de empresas se hace referencia al "administrador", "director", "gerente”, etc. Mostrando desde allí un dominio masculino en las organizaciones.

Estructuras jerárquicas "neutras". Centrarse solo en la estructura organizacional implica no tomar en cuenta al individuo, ni sus motivaciones, así pues, la asignación de tareas, el ejercicio de la autoridad y la coordinación de funciones son actividades que constituyen la estructura de una organización. Aunque, se reconoce que de alguna forma impacta la diferencia sexual en la socialización, el ejercicio cotidiano del trabajo y expresiones de los patrones de control ejercidos. Sin embargo, no se admite que el ejercicio de los mecanismos de control con que cuenta toda organización pueda variar según el género de los individuos, ya que coexiste la regla formal y la cultural basada en el dominio masculino.

Esta propuesta ha permitido reflexionar la conformación de otras líneas de investigación en los últimos cinco años y que han sido presentadas en trabajos de dos Congresos Internacionales de administración en México, los cuales se presentan a continuación:

Tabla 1. Líneas de investigación de estudios de género en las organizaciones

\begin{tabular}{|c|c|}
\hline Temas & Autores \\
\hline $\begin{array}{c}\text { Emprendimiento Femenino y } \\
\text { Empresarias }\end{array}$ & $\begin{array}{l}\text { Zabludovsky y Avelar (2001), Zabludovsky (2003), Izquierdo y } \\
\text { Schuster (2008), Camarena y Saavedra (2015a), Escamilla, Z., Ca- } \\
\text { rrillo, S. y Caldera, D. (2011), Escamilla, Z., Caldera, D. y Ortega, M. } \\
\text { (2012), Vidal, J., Mafud, M. y Pérez, J. (2013), Riveros, A., Rosales, } \\
\text { T. y Candelario, J. (2015), Chávez, I. y Álvarez, L. (2015), Camare- } \\
\text { na, M., Saavedra, M. y Hernández, L. (2015), Briceño, O. y Briceño, } \\
\text { A. (2011), Vanarro, G. y Camacho C. (2011), Arredondo, F., Maldo- } \\
\text { nado, V. y Rosas J.A. (2011), Macías, G., Dichiaria, G. y García, G. } \\
\text { (2012), Chauca, P. y Gonzáles, M. (2013), Vargas, J. (2013), Lino, } \\
\text { J., Cárdenas, A. y López, S. (2014), Kuri, E., Gutiérrez, F. y Flores, J. } \\
\text { (2014). }\end{array}$ \\
\hline Liderazgo Femenino & $\begin{array}{l}\text { Diez, Valle, Terrón y Centeno (2003), Zabludovsky (2002), Za- } \\
\text { bludovsky (1997), Carranza, G., Muro, José y Coronado, J. (2011), } \\
\text { Moncayo, B. y Villalba, C. (2013), Del Pino, R. (2013), Trujillo, M., } \\
\text { Valderrábano, M. y Lámbarry, F. (2013), Moncayo, B. y Zuluaga, D. } \\
\text { (2014a), Moncayo, B. y Zuluaga, D. (2014b), Moncayo, B. y Zulua- } \\
\text { ga, D. (2015), Fischer, L. y Ursul, J. (2015), Cánovas, C. y Vázquez, } \\
\text { M. (2014), Velázquez, L., Arredondo, F. y Hernández, L. (2015), } \\
\text { Gutiérrez, M., García, M. y Reyes, J. (2014). }\end{array}$ \\
\hline Equidad de género en el trabajo & $\begin{array}{c}\text { (Piazze, 2009), De la Cruz, N. (2013), Martínez, P., Escobedo, H. y } \\
\text { García, M. (2015), Ibarvo, V., Jiménez, G. y Granillo, L. (2015), Mar- } \\
\text { tínez, C., Romo, L., y Rangel, P. (2011), Colín, M., Rodríguez, R. y } \\
\text { Velázquez, N. (2012), Madrigal, B., Marún, E. y Madrigal, R. (2013), } \\
\text { Ricalde, G., Sosa, P. y Mejía, N. (2013), Verján, R. y Díaz, I. (2014), } \\
\text { Macías, G. (2015). }\end{array}$ \\
\hline Capital humano con enfoque de género & $\begin{array}{c}\text { Arredondo, F. y De la Garza, J. (2012), Izquierdo, B. y Montano J. } \\
\text { (2012), Juárez, S., Izquierdo, B. y Viñán, Hernández, H., Garrido, P. } \\
\text { y Rico, M. (2015) A. (2015), Izquierdo, B. (2012), Balam, E., Canto, } \\
\text { A. y Aguilar, R. (2013). }\end{array}$ \\
\hline
\end{tabular}




\begin{tabular}{|c|c|}
\hline Temas & Autores \\
\hline $\begin{array}{l}\text { El género en el estudio de las organizacio- } \\
\text { nes }\end{array}$ & $\begin{array}{l}\text { Vázquez, A. (2013), Jiménez, D. y Beltrán, J. (2015), Quiroz, M. } \\
\text { (2015), Arredondo, F. y Villareal, M. (2013), Salaiza, F., Montoya, N. } \\
\text { y López, S. (2015). }\end{array}$ \\
\hline Techo de Cristal & $\begin{array}{c}\text { Camarena, Saavedra y Ducloux (2015b), Colín, M., Virginia, C. y } \\
\text { Granados, L. (2011), Aguilar, R. y Pinzón, L. (2011), Cuevas, V., } \\
\text { Arango, A. y Reyna, V. (2012). }\end{array}$ \\
\hline Género y Mobbing & $\begin{array}{l}\text { Trujillo, M., Valderrábano, M. y Lámbarry, F. (2012), Del Pino, R. } \\
\text { (2014). }\end{array}$ \\
\hline Mercadotecnia y género & $\begin{array}{c}\text { Jiménez, O. y Ojeda, R. (2015), Sahui, J. (2012), Torres, F., Cubillo, } \\
\text { G. y Olivares, M. (2014). }\end{array}$ \\
\hline $\begin{array}{l}\text { La mujer en los consejos de administración } \\
\text { de las empresas }\end{array}$ & $\begin{array}{l}\text { Jimeno de la Maza, F., Redondo, M. y Zárate, M., Zárate, M., Redon- } \\
\text { do, M. y Jimeno, F. (2014). }\end{array}$ \\
\hline Redes sociales y desarrollo de la mujer & $\begin{array}{c}\text { Colín, M., Gallaga, M.E. y Conraud, E. (2012), Arredondo, F., Veláz- } \\
\text { quez, L. y De la Garza, J. (2014). }\end{array}$ \\
\hline Competencias con enfoque de género & Quevedo, L., Espericueta, D. y Méndez, M. (2012). \\
\hline Género, ética y RSE & Romo, L., Leal, F. y Carrillo, I. (2014). \\
\hline Formación emprendedora de la mujer & Macías, G., Romo, L. y Huerta, E. (2014). \\
\hline Emprendedoras rurales & Chong, E., Santamaría, E. y Díaz, M. (2014). \\
\hline Trabajo, familia y desarrollo profesional & Garrido, N., Garibay, M. y Reza, J. (2015). \\
\hline La mujer en la empresa familiar & Velandia, M. y Herrera, N. (2015). \\
\hline Mujeres y redes de conocimiento & Cárdenas, M. (2015). \\
\hline
\end{tabular}

Fuente: Elaboración propia con base en los autores citados y las memorias del XV, XVI, XVII, XVIII, y XIX, Congreso Internacional de Ciencias Administrativas, ACACIA, A.C. (2011-2015), así como las memorias del XVI, XVII, XVIII, XIX y XX, Congreso de administración, Contaduría e Informática de la Facultad de Contaduría y Administración de la Universidad Nacional Autónoma de México (2011-2015).

De acuerdo con la revisión realizada de las investigaciones que se han presentado en los últimos cinco años, se puede observar que uno de los temas más investigados es el de empresarias y emprendimiento femenino, el segundo aborda el liderazgo femenino, equidad de género en el trabajo ocupa el tercer lugar.

Lo anterior, muestra la preocupación de los investigadores mexicanos por las limitaciones que encuentra la mujer en el ámbito laboral para ascender en sus carreras, muchas mujeres emprenden al encontrar en el desarrollo de sus carreras un techo de cristal que les impide ascender de puesto, por otro lado, se encuentran los estudios que se han ocupado del tema de liderazgo femenino, donde las mujeres, 
tanto en entidades públicas como privadas, tienen que salvar barreras que han estereotipado su actuación, la equidad de género en el trabajo es otro de los aspectos que dan cuenta de la brecha que aún existe en las organizaciones, en el trato a hombres y mujeres.

Mientras que el techo de cristal, el capital humano con enfoque de género y el género en el estudio de las organizaciones ocupan el cuarto lugar de investigaciones realizadas, sumándose a la preocupación por la equidad de género en las organizaciones y por la forma en que se puede incorporar la perspectiva de género en el estudio de las organizaciones en el ámbito de las ciencias contable administrativas.

Se observa que los investigadores han puesto menos énfasis en temas como: género y mobbing, mercadotecnia y género, mujeres y redes de conocimiento, la mujer en la empresa familiar la mujer en los consejos de administración de las empresas, competencias con enfoque de género, redes sociales y desarrollo de la mujer, género ético y responsabilidad social empresarial, formación de la mujer emprendedora, emprendedoras rurales y trabajo, familia y desarrollo profesional. Denotando con esto que los estudios de género en el ámbito de las organizaciones es un tema emergente en el campo de las ciencias administrativas, pues a diferencia de otros temas más ampliamente estudiados, los trabajos encontrados en estos dos importantes Foros de investigación son pocos, por lo que este tema que debe ser fomentado para incorporar la perspectiva de género en el currículo de estudios de los programas de las licenciaturas en ciencias contable administrativas en México.

\section{Propuesta para incorporar en el currículo universitario de las carreras contables administrativas los estudios de género en las organizaciones}

Existen dificultades que hacen complicada la superación de los estereotipos y el avance hacia la igualdad y respeto. Algunas de las que se consideran más importantes y que hacen lento y tortuoso el avance educativo de la escuela son las siguientes (Santos, 1997: 17):

a. La escuela es una reproducción de la sociedad ya que está integrada por personas que se encuentran inmersas en ella. No es posible abandonar en la puerta de la escuela los estereotipos que se tienen en la vida. Además, la escuela es una institución que mantiene y perpetúa las estructuras y el funcionamiento de la sociedad, marcadamente androcéntricos.

b. El individualismo que impide un planteamiento colegiado de los procesos educativos. La balcanización que sufre la actividad escolar (un profesor, un grupo, un lugar, una materia, etc.) hace difícil la implantación del paradigma de la colegialidad ${ }^{12}$. La fragmentación de los planteamientos, de las acciones educativas y de los mecanismos de evaluación institucional merma la eficacia de la tarea.

c. La rutinización de los comportamientos y de las actitudes y concepciones de los profesionales hacen difícil la reflexión y la autocrítica institucional. La escuela es una institución que cambia con dificultad, ya, que tiene una fuerte presión social y, goza de escasa autonomía.

12- La colegialidad exige concepciones compartidas que permitan entender, planificar, desarrollar y evaluar de forma cohesionada y coherente una institución. 
d. El arraigo de los estereotipos de género, profundamente implantados en el currículum oculto de los docentes. La preponderancia del pensamiento hegemónico, de carácter androcéntrico, que de forma más o menos burda, preside y hegemoniza la vida de la universidad.

e. La prioridad que se concede en el currículum explícito a los contenidos científicos de las diversas materias. Contenidos que están impregnados (en su selección, en su estructura y en su misma epistemología) por elementos de carácter discriminatorio. La pretendida neutralidad de la ciencia y de los contenidos que se seleccionan de ella para configurar el currículum básico es un peligro que impide la crítica y el cambio.

Una de las razones por las que no se han superado los estereotipos de género tendría que ver con el desconocimiento de los estudiantes universitarios acerca de las leyes que defienden los derechos de la mujer, ya que en su opinión existen prácticas discriminatorias (Ojeda y Avilés, 2005: 15).

En este sentido, en relación con el papel de las IES, cabe preguntarse de qué manera la educación superior puede favorecer la transformación de la cultura machista que afecta la calidad de vida de las personas, es así como surge el cuestionamiento sobre como introducir el tema de la perspectiva de género en las aulas universitarias y en el caso que nos ocupa en las Facultades y Escuelas de Contaduría y Administración, de manera que se logre transformar los conceptos y comportamientos de los jóvenes en la familia, en el trabajo y en el conjunto de la sociedad. Se considera que esto es importante, porque una característica del perfil de los egresados de muchas facultades es ejercer un liderazgo en las organizaciones de manera que en su actuar busque la equidad de género (Ojeda y Avilés, 2005: 25).

A este respecto Bouquet (2011) señala que cuando se plantea que deben incorporarse materias de género en todas las carreras, no se busca la especialización de las nuevas generaciones en temas de género, sino que el alumnado conozca esta perspectiva de análisis dentro de la disciplina en la que se está formando, ya que le dará una gran cantidad de herramientas para el análisis crítico de los propios paradigmas de su disciplina.

A manera de ejemplo se presenta el impacto que lo anterior puede tener, considerando tres carreras: derecho economía y psicología.

\section{Tabla 2. La incorporación de la perspectiva de género} en las carreras de derecho, economía, psicología y administración

\begin{tabular}{|c|c|}
\hline Carrera & Razones para incorporar de la perspectiva de género \\
\hline Derecho & $\begin{array}{l}\text { El desconocimiento de la precariedad del estatus legal de las mujeres durante siglos y } \\
\text { cómo éste se fue reconstruyendo, al no abordar los acuerdos internacionales en materia } \\
\text { de igualdad, o ignorar el trato diferenciado que se da en los ministerios públicos a } \\
\text { hombres y a mujeres, así como la falta de marcos legales claros y equitativos, para la } \\
\text { protección de los derechos de las mujeres ante situaciones de acoso y violencia sexual en } \\
\text { el ámbito laboral o familiar, lo deja con una formación incompleta y sesgada. }\end{array}$ \\
\hline Economía & $\begin{array}{c}\text { El conocimiento del origen - o la arbitrariedad-de la división sexual del trabajo y la } \\
\text { separación de las economías productiva y reproductiva, donde a la primera se le otorga } \\
\text { primacía sobre la segunda, imprime nuevos cuestionamientos a desigualdades y proble- } \\
\text { mas de orden económico que deben ser debatidos en esta disciplina a fin de proponer } \\
\text { soluciones a esta problemática social. }\end{array}$ \\
\hline
\end{tabular}


- Revista de Ciencias Sociales y Humanidades. ISSN-P: 0188-9834 ISSN-E: 2395-8669.

\begin{tabular}{|c|c|}
\hline Carrera & \multicolumn{1}{|c|}{ Razones para incorporar de la perspectiva de género } \\
\hline Psicología & $\begin{array}{c}\text { La constitución de los sujetos y sus identidades está atravesada por un ordenamiento de } \\
\text { género que configura psiques diferenciadas desde una estructura cultural, en la que inter- } \\
\text { viene un orden simbólico, un imaginario y procesos subjetivos que definen y determinan } \\
\text { distintas formas de personalidad entre los sexos, por lo que el conocimiento puntual de } \\
\text { estas diferencias permite preparar e implementar esquemas de tratamiento distintos, } \\
\text { considerando las diferencias. }\end{array}$ \\
\hline Administración & $\begin{array}{c}\text { Debe reconocer la hegemonía masculina en la dinámica organizacional, esto se da como } \\
\text { consecuencia de que la literatura en administración ha sido creada en torno al modelo } \\
\text { analítico hegemónico de organizaciones y ejercicio de la administración, con una clara do- } \\
\text { minación masculina. De donde se desprenden anarquías organizadas que caracterizan al } \\
\text { tomador de decisiones, al estratega, es decir funciones importantes en donde no ha sido } \\
\text { incorporada la perspectiva de género. En toda la literatura sobre gestión de empresas } \\
\text { se hace referencia al "administrador", “director", “gerente”, etc. Mostrando desde allí un } \\
\text { dominio masculino en las organizaciones. }\end{array}$ \\
\hline
\end{tabular}

Fuente: Elaboración propia con base en Buquet (2011) y Jiménez y Beltrán (2015).

A este respecto, Mora y Pujal (2009) señalan que la introducción de la perspectiva de género en la docencia universitaria debe realizarse revisando a fondo no sólo las competencias propias de la asignatura sino el sesgo de género y la oportunidad de introducir ciertas perspectivas de género que tengan un significado consistente con la carrera a la que pertenece la asignatura.

Por lo tanto, resulta relevante incorporar la perspectiva de género en el currículo, así como en planes y programas de estudio, creando materias específicas, temas y contenidos sobre los estudios de género en las diferentes áreas del conocimiento (Cervantes, 2009: 123).

Se considera que los temas que podrían incluirse los programas de estudios de las licenciaturas contables administrativas, deben ser:

- Empresarias y emprendimiento femenino

- Liderazgo femenino

- Equidad de género en el trabajo

- Techo de cristal

- Capital humano con enfoque de género

- El género en el estudio de las organizaciones

- Género y mobbing

- Mercadotecnia y género

- Mujeres en la empresa familiar

- La mujer en los consejos de administración de las empresas

- Capacitación en empresas dirigidas por mujeres

- Competencias con enfoque de género 
- Redes sociales y desarrollo de la mujer

- Género ético y responsabilidad social empresarial

- Formación de la mujer emprendedora

- Trabajo, familia y desarrollo profesional

- La perspectiva de género en la cultura organizacional

- Hegemonía masculina en la dinámica organizacional

Estos temas son enunciativos, más no limitativos, dado que se puede incorporar la perspectiva de género en todas las temáticas que abordan el estudio de las organizaciones el campo de la administración.

En el caso de la materia Empresarias y emprendimiento femenino, se podría configurar con las temáticas siguientes: El entorno empresarial mexicano y su impacto en las empresas lideradas por mujeres, empoderamiento femenino, características de las empresas lideradas por mujeres, capital humano en las empresas lideradas por mujeres, financiamiento en las empresas lideradas por mujeres, redes empresariales y su influencia en las empresas lideradas por mujeres. Lo anterior a modo de ejemplo, el contenido de las materias dependerá de la disponibilidad de estudios que existan en torno al tema y el enfoque que los diseñadores de los programas le quieran dar a las materias.

\section{Conclusiones}

Los estudios de género en las organizaciones permiten analizar cómo se puede incorporar la perspectiva de género en el ámbito laboral, reconociendo que hombres y mujeres enfocan su desarrollo y promoción profesional de manera diferente, tienen diferentes estilos de liderazgo, y diferentes enfoques al dirigir su propia empresa. A pesar de la importancia que tienen los estudios de género para impulsar políticas, programas y proyectos de desarrollo que fomenten la equidad de género, estos son incipientes en el contexto mexicano. Aunque es necesario destacar que en el gobierno actual por primera vez se incorpora la perspectiva de género en el Plan Nacional de Desarrollo 2013-2018 y en el Programa Sectorial de Educación Pública 2013-2018:

El camino para incorporar los estudios de género en el currículo universitario ha sido largo, pues inicio en los años setenta y en la actualidad aún no se ha logrado implementar de manera formal y definitiva en todos los programas de estudio, dejando ver con esto que hay mucho todavía por hacer en este tópico.

Las investigaciones acerca de los estudios de género en las organizaciones, dan cuenta de la preocupación que existe por parte de la comunidad académica acerca de la inequidad de género, hecho que resalta la importancia de incorporar el enfoque de género en el currículo de los programas de las licenciaturas en ciencias contable administrativas, dado que la característica de los egresados de estas carreras es ejercer una posición de liderazgo en las organizaciones, muchas de las cuales hoy en día no han logrado superar los estereotipos de género.

Dentro de las temáticas que se propone sean incorporadas en el currículo de los programas de las licenciaturas en ciencias contable administrativas destaca el estudio de empresarias y emprendimiento femenino, tema escasamente estudiado, toda vez que la participación de la mujer como dueña de empresa va en ascenso en México y es necesario realizar estudios en este tópico, para conocer su problemática 
y proponer soluciones acordes con su realidad. Otro tema a destacar trata el aspecto de la hegemonía masculina en la dinámica organizacional, el cual es un campo de conocimiento de gran importancia, pues no existen estudios que muestren un diagnóstico de la situación actual en el contexto mexicano y que permita ser un impulsor de futuros estudios.

\section{Referencias}

ACACIA. 2011. Memorias del XV Congreso internacional de la Academia de Ciencias Administrativas, ACACIA, A.C., México.

ACACIA. 2012. Memorias del XVI Congreso internacional de la Academia de Ciencias Administrativas, ACACIA, A.C., Veracruz, Veracruz.

ACACIA. 2013. Memorias del XVII Congreso internacional de la Academia de Ciencias Administrativas, ACACIA, A.C., Guadalajara.

ACACIA. 2014. Memorias del XVIII Congreso internacional de la Academia de Ciencias Administrativas, ACACIA, A.C., Tijuana.

ACACIA. 2015. Memorias del XIX Congreso internacional de la Academia de Ciencias Administrativas, ACACIA, A.C., Durango, Durango.

Bolaños, Carolina. 2003. Curriculum universitario, género sensitivo e inclusivo. Ciencias Sociales 101102: 71-78.

Buquet, Ana. 2011. Transversalización de la perspectiva de género en la educación superior. Problemas conceptuales y prácticos. Perfiles Educativos, 9(33): 211-225.

Caballero, Rebeca. 2011. El diseño curricular como estrategia para la incorporación de la perspectiva de género en la educación superior. Revista Latinoamericana de estudios educativos, 41(3-4): 45-64.

Camarena, María y Hernández, Cleotilde. 2005. Feminismo y organizaciones una propuesta de equidad. Ponencia presentada en el IX Congreso Anual de la Academia de Ciencias Administrativas, ACACIA, Mérida.

Camarena, María y María Saavedra. 2015a. Diferencias en la competitividad de las empresas según el género del director. Neumann Business Review, 1(2): 70-86.

Camarena, María, María Saavedra y Daniela Ducloux. 2015b. El techo de cristal y la situación de las mujeres en los puestos directivos en México. Ponencia presentada en el XX Congreso Internacional de la Academia de Ciencias Administrativas, ACACIA, A.C, Mérida.

Camarena, María, María Saavedra y Daniela Ducloux. 2015c. Un panorama del género en México: Situaciónactual.Revista Guillermo de Ockham, 13(2):77-87.DOI:http://dx.doi.org/10.21500/22563202.2066 Cardaci, Dora.2005. ¿Ausentes o invisibles? Contenidos sobre las mujeres y los géneros en el currículo de licenciatura de universidades mexicanas. La Ventana, 3(21): 107-142.

Cervantes, Celia. 2009. Los estudios de la mujer y de género en México y las redes académicas de coordinación Interinstitucional. En Estudios de género y desarrollo. Balance y propuestas, coordinado por Estefania y Abad Nava. España: Universidad Autónoma de Madrid, 95-130.

CIM. 2011. Avance de la igualdad de género en el marco del trabajo decente Washington: Comisión Interamericana de Mujeres (CIM), Organización de Estados Americanos. 
Diez, Enrique, Rosa Valle, Eloína Terrón y Begoña Centeno. 2003. El liderazgo femenino y su ejercicio en las organizaciones educativas. Revista Iberoamericana de Educación, (31): 1-19.

Durán, Martha. 2012. La transversalidad de género en la educación superior: propuesta de un modelo de implementación, Revista Posgrado y Sociedad, 12 (1): 23-43.

FCA-UNAM. 2011. Memorias del XVI Congreso Internacional de Contaduría Administración e informática, México.

FCA-UNAM. 2012. Memorias del XVII Congreso Internacional de Contaduría Administración e informática, México.

FCA-UNAM. 2013. Memorias del XVIII Congreso Internacional de Contaduría Administración e informática, México.

FCA-UNAM. 2014. Memorias del XIX Congreso Internacional de Contaduría Administración e informática, México.

FCA-UNAM. 2015. Memorias del XX Congreso Internacional de Contaduría Administración e informática, México.

Flores, Aurelia, María Carrasco y Adelina Espejel. 2014. La formación de la comisión universitaria para la igualdad de género (CUIG). Una experiencia de transversalización. Ra Ximhai, 10(7): 23-36.

González, Rosa. 2009. Estudios de género en educación. Una rápida mirada. Revista Mexicana de Investigación Educativa, 14 (42): 681-699.

Gobierno de la Republica de México. (2013). Plan Nacional de Desarrollo 2013-2018. Recuperado de http://pnd.gob.mx

Heller, Lidia. 2010. Mujeres emprendedoras en América Latina y el Caribe: realidades, obstáculos y desafíos. Santiago de Chile: Naciones Unidas-CEPAL.

Izquierdo, Belinda y Juan Schuster. 2008. Construcción de indicadores para la competitividad. Caso de empresas dirigidas por mujeres en la región de Xalapa, Ver. México. Investigación Administrativa, 37 (102): 82-102.

Jiménez, Diana y Jesús Beltrán. 2015. Análisis de la creación de la teoría administrativa desde una perspectiva de género. Ponencia presentada en el XX Congreso Internacional de Contaduría, Administración e Informática, México.

Maceira, Luz. 2005. Investigación del currículo oculto en la educación superior: alternativa para superar el sexismo en la escuela. La Ventana, 3(21): 187-227.

Marinsalta, Claudia. 2010. Cuestionando las ausencias en el currículo universitario. Congreso Internacional: Las políticas de equidad de género en prospectiva: nuevos escenarios, actores y articulaciones, Buenos Aires, Noviembre.

Molina, Estefanía y Abad Nava. 2009. Estudios de género y desarrollo. Balance y propuestas. Cuadernos Solidarios $\mathrm{N}^{\circ}$ 5. España: Universidad Autónoma de Madrid.

Mora, Enrico y Margot Pujal. 2009. Introducción de la perspectiva de género en la docencia universitaria. II Congreso Internacional 'Claves para la implicación de los estudiantes en la Universidad', Gerona.

Moreira, Claudia. 2010. Liderazgo transformacional y género en organizaciones militares. Tesis Doctoral. Madrid, España: Universidad Complutense de Madrid.

Mosteiro, Josefa. 1997. El género como factor condicionante de la elección de carrera: Hacia una ori- 
entación para la igualdad de oportunidades entre los sexos. Revista galego-portuguesa de psicoloxía e educación, 1(1): 305-315.

Navarro, Natalia. 2000. Género: una perspectiva intraorganizacional en política de género y gestión de ONGD. España: ACSUR-Las Segovias.

Navarro, Natalia. 2003. Cambiar el Chip: cómo promover cambios en pro equidad de género en las organizaciones. España: ACSUR-Las Segovias.

Navarro, Natalia. 2007. Desigualdades de género en las organizaciones: Procesos de cambio organizacional pro equidad. San Salvador: Programa de las Naciones Unidas para el Desarrollo.

OIT. 2011. La transversalidad de género. Módulo para la formación de mujeres y hombres sindicalistas. Turín: Centro Internacional de Formación de la Organización Internacional del Trabajo.

Ojeda, Ruth y Avilés, Carolina. 2005. Los derechos humanos en la curricula de los programas de maestría en la FCA de la UADY. Presentado en las memorias del IX Congreso Anual de la Academia de Ciencias Administrativas, ACACIA, Mérida.

Piazze, Ada. 2009. Género y negocios: casos exitosos en cuatro continentes. Banco Interamericano de Desarrollo.

Rivera, Elsa. 2003. Género y Universidad. Una aproximación al estudio de las académicas de la Benemérita Universidad Autónoma de Puebla. Colección Pedagógica Universitaria, 40: 1-18.

Santos Sanz, María. 1994. La mujer y la dirección escolar. Organización y gestión educativa, 11: 7-10.

Santos, Miguel. 1997. El curriculum oculto y la construcción del género en la escuela. Kikiriki. Cooperación educativa, 2(42-43): 14-27.

Secretaria de Educación Pública. 2013. Programa sectorial de educación 2013-2018. México: SEP.

Serret Estela y Jessica Méndez. 2011. Sexo, género y feminismo. México: Suprema Corte de Justicia de la Nación, Tribunal Electoral del Poder Judicial de la Federación, Instituto Electoral del Distrito Federal.

Serret, Estela. 2008. Qué es y para qué es la perspectiva de género. Oaxaca, México: Instituto de la Mujer Oaxaqueña.

Vázquez, Ángel. 2013. Los estudios de género en el estudio de las organizacionales. Posibilidades de análisis en México. Ponencia presentada en el XVIII Congreso Internacional de Contaduría, Administración e Informática, D.F.

Velasco, Ivonne. 2007. Hacia una educación superior con equidad de género. Inventio la génesis de la cultura universitaria en Morelos, 3(5): 43-48.

Watzlawick, Paul. 1989. El arte de amargarse la vida. Barcelona: Editorial Herder, S.A.

Zabludovsky Gina, Sonia de Avelar. 2001. Empresarias y ejecutivas en México y Brasil. Facultad de Ciencias Políticas y Sociales. México: UNAM.

Zabludovsky Gina. 2002. Las políticas de la diversidad y las mujeres en las grandes compañías privadas en México, en Mujeres en cargos de dirección en América Latina, estudios sobre Argentina Chile, México y Venezuela. Miguel Ángel Porrúa, UNAM, México D.F.

Zabludovsky, Gina. 1997. Presencia de las mujeres ejecutivas en México. Sociológica, (33): 73-97.

Zabludovsky, Gina. 2003. Las empresarias, sus familias y sus sociedades. Comercio exterior, 2 (62): 18-24. 